\title{
PPM Performance with BWT Complexity: A Fast and Effective Data Compression Algorithm
}

\author{
MICHELLE EFFROS
}

This paper introduces a new data compression algorithm. The goal underlying this new code design is to achieve a single lossless compression algorithm with the excellent compression ratios of the Prediction by Partial Mapping (PPM) algorithms and the low complexity of codes based on the Burrows Wheeler Transform (BWT). Like the BWT-based codes, the proposed algorithm requires worst case $O(n)$ computational complexity and memory; in contrast, the unbounded-context PPM algorithm, called PPM ${ }^{*}$, requires worst case $O\left(n^{2}\right)$ computational complexity. Like PPM*, the proposed algorithm allows the use of unbounded contexts. Using standard data sets for comparison, the proposed algorithm achieves compression performance better than that of the BWT-based codes and comparable to that of $P P M^{*}$. In particular, the proposed algorithm yields an average rate of 2.29 bits per character (bpc) on the Calgary corpus; this result compares favorably with the 2.33 and 2.34 bpc of PPM5 and PPM* (PPM algorithms), the 2.43 bpc of BW94 (the original BWT-based code), and the 3.64 and $2.69 \mathrm{bpc}$ of compress and gzip (popular Unix compression algorithms based on Lempel-Ziv(LZ) coding techniques) on the same data set. The given code does not, however, match the best reported compression performance-2.12 bpc with PPMZ9-listed on the Calgary corpus results web page at the time of this publication. Results on the Canterbury corpus give a similar relative standing. The proposed algorithm gives an average rate of $2.15 \mathrm{bpc}$ on the Canterbury corpus, while the Canterbury corpus web page gives average rates of 1.99 bpc for PPMZ9, 2.11 bpc for PPM5, 2.15 bpc for PPM7, 2.23 bpc for BZIP2 (a popular BWT-based code), and 3.31 and $2.53 \mathrm{bpc}$ for compress and gzip, respectively.

Keywords-Burrows Wheeler Transform, lossless source coding, prediction by partial mapping algorithm, suffix trees, text compression.

\section{INTRODUCTION}

The field of lossless data compression (also known as "text compression" despite its wider applicability) is undergoing a fascinating period of development. The distance between theory and practice and the difference between the codes with the best compression performance and the codes with the best complexity performance are shrinking at dramatic rates. This paper pursues the second of these two goals, achieving

Manuscript received February 15, 2000; revised August 8, 2000. This work was partially supported by NSF Award CCR-9909026.

The author is with the Department of Electrical Engineering, 136-93, California Institute of Technology, Pasadena, CA 91125 USA (e-mail: effros@caltech.edu).

Publisher Item Identifier S 0018-9219(00)09984-9. the compression performance of the Prediction by Partial Mapping (PPM) algorithms and the complexity of codes based on the Burrows Wheeler Transform (BWT) in a single lossless code.

The PPM algorithm and its descendants (e.g., [1], [2]) are extremely effective data compression techniques. In tests on standard data sets such as the Calgary and Canterbury corpora, ${ }^{1}$ the performance of PPM codes consistently meets or exceeds the performance of a wide array of algorithms. For example, PPM algorithms give significant performance improvements over the popular Lempel-Ziv (LZ) algorithms LZ77 [5], LZ78 [6], LZW [7] and their descendants. In particular, using the Calgary corpus for comparison, the unbounded context PPM algorithm (PPM*) gives compressed file sizes (measured in bits per character, or bpc) that are, on average, $35.7 \%$ smaller than those achieved by the UNIX compression utility compress. Likewise, $\mathrm{PPM}^{*}$ gives file sizes that are, on average, $13 \%$ smaller than those of $g z i p$. Both compress and gzip are LZ codes.

PPM algorithms achieve their excellent performance using a collection of probability models. These models describe the conditional probability distribution on each upcoming symbol in the data sequence given the "context" provided by the symbols that came before. The models in the collection correspond to contexts of different lengths. PPM algorithms differ from earlier context-modeling approaches in their particular methods for adaptively designing the collection of models and combining the members of the collection to obtain a single model for use in coding.

Despite their superior compression performance, PPM codes are far less commonly applied than algorithms like LZ77, LZ78, and LZW. In practice, the LZ codes are favored over PPM algorithms for their relative efficiencies in memory and computational complexity [3]. While the LZ codes can be implemented with $O(n)$ memory and complexity (e.g., [8]), straightforward implementations of some PPM algorithms require worst case $O\left(n^{2}\right)$ memory and complexity to code a data sequence of length $n$. While $\mathrm{PPM}^{*}$ implementations requiring only $O(n)$ memory have

\footnotetext{
${ }^{1}$ The Calgary [3] and Canterbury [4] corpora are standard data sets used for comparing the experimental performance of lossless compression algorithms. Each data set contains a variety of data types. Examples include text files (both in English and in a variety of programming languages), a CCITT fax test set, an Excel spreadsheet, and a SPARC executable.
} 


\begin{tabular}{|c||ccccccc|}
\hline 1 & $\mathrm{a}$ & $\mathrm{n}$ & $\mathrm{a}$ & $\mathbf{n}$ & $\mathrm{a}$ & $\mathrm{s}$ & $\mathrm{b}$ \\
\hline 2 & $\mathrm{a}$ & $\mathrm{n}$ & $\mathrm{a}$ & $\mathrm{s}$ & $\mathrm{b}$ & $\mathrm{a}$ & $\mathrm{n}$ \\
\hline 3 & $\mathrm{a}$ & $\mathrm{s}$ & $\mathrm{b}$ & $\mathrm{a}$ & $\mathrm{n}$ & $\mathrm{a}$ & $\mathrm{n}$ \\
\hline 4 & $\mathbf{b}$ & $\mathbf{a}$ & $\mathbf{n}$ & $\mathbf{a}$ & $\mathbf{n}$ & $\mathbf{a}$ & $\mathbf{s}$ \\
\hline 5 & $\mathrm{n}$ & $\mathrm{a}$ & $\mathrm{n}$ & $\mathrm{a}$ & $\mathrm{s}$ & $\mathrm{b}$ & $\mathrm{a}$ \\
\hline 6 & n & $\mathrm{a}$ & $\mathrm{s}$ & $\mathrm{b}$ & $\mathrm{a}$ & $\mathbf{n}$ & $\mathrm{a}$ \\
\hline 7 & $\mathrm{~s}$ & $\mathrm{~b}$ & $\mathrm{a}$ & $\mathrm{n}$ & $\mathrm{a}$ & $\mathbf{n}$ & $\mathrm{a}$ \\
\hline
\end{tabular}

Fig. 1. The BWT of the sequence "bananas". The rows of the above table are the 7 cyclic shifts of the sequence "bananas". The rows are ordered alphabetically. The original data sequence (in bold) appears in row 4 . The final column of the table contains the sequence "bnnsaaa". Hence, BWT (bananas) = (bnnsaaa, 4).

been proposed in the literature [2], [9]-[11], $O\left(n^{2}\right)$ worst case complexity is inherent to the $\mathrm{PPM}^{*}$ algorithm [11]. The high computational complexity of PPM algorithms remains an impediment for their more widespread use.

In recent years, lossless data compression algorithms based on the BWT [12] have become increasingly popular both in the literature and in practice. This increasing popularity arises from the fact that BWT-based codes partially span the performance gap between the PPM and LZ algorithms without simultaneously spanning the gap in complexity. That is, BWT-based codes require computational complexity comparable to that of the LZ algorithms but yield significant performance improvements over popular LZ codes [2], [13], [14]. For example, the original BWT compression algorithm [12], here called BW94, gives a 33\% improvement in average file size over compress and a $9.7 \%$ improvement in average file size over gzip on the Calgary corpus. These results go a long way toward closing the $35.7 \%$ performance gap between $\mathrm{PPM}^{*}$ and compress and the $13 \%$ performance gap between PPM $^{*}$ and gzip on the same data set.

BWT-based codes are conceptually simple. The encoder transforms the data sequence with the BWT and then compresses the transform output with a very simple code. The decoder reverses this process, first decoding the transformed data and then performing the inverse transform. ${ }^{2}$

The BWT output includes a permuted copy of the input data sequence and a single integer. Intuitively, the BWT may be found by creating a table in which each row is a distinct cyclic shift of the original data sequence. The rows are ordered lexicographically. The BWT output contains the sequence found in the table's last column and the integer describing the row containing the original data sequence. An example appears in Fig. 1. Since the last character of a given row is the character that precedes that row's first character in the original data sequence, and since the lexicographical ordering of the rows places all rows that begin with the same string consecutively, the BWT groups together all symbols that precede the same (arbitrary length) string. As a result, the BWT of a reversed data sequence groups together all symbols that follow a given string - that is, all symbols with the same unbounded context.

The BWT output is well-suited for compression. Combining a simple lossless compression algorithm with a

${ }^{2}$ The BWT is reversible - any data sequence may be uniquely derived from its transform. The inverse BWT is described in [12]. low-complexity transform implementation yields a family of $O(n)$ complexity and memory lossless codes with very good experimental compression performance (e.g., [12]-[14]). Theoretical investigation of the BWT output leads to a family of low-complexity universal lossless source codes for stationary ergodic sources [15], [16].

While the best of the above-mentioned universal codes converges to the optimal performance at a rate within a constant factor of the optimal rate of convergence theoretically achievable on finite-memory sources [15], [16], the performance of these codes on finite sequences from sources such as text fails to meet the performance of competing codes such as the PPM algorithms [17]. This failure arises both from inaccuracies in the source model and from the fact that the constants - even the constants on lower order terms in the rate of convergence - can have a large impact on performance results on finite-length files.

BWT-based codes, which code all symbols with the same context consecutively and, therefore, track only one probability model at a time, achieve significant complexity savings relative to PPM algorithms, which adapt and store probability models for all contexts simultaneously. Yet this savings comes at a price. By coding symbols in their original order, PPM codes allow both encoder and decoder to track the context information. (The context of the next symbol is given by the symbols that come before.) The use of the BWT results in a nonsequential code and a decoder that cannot independently track the state information.

Modifying the BWT-based codes of [15]-[17] in order to make the context information accessible to the decoder yields the new low-complexity, low-memory data compression algorithm introduced in [18] and described in detail here. The algorithm combines computational efficiencies inspired by BWT-based codes with sequential coding and unbounded context source modeling techniques reminiscent of PPM* The proposed algorithm can be described as a variation on either BWT-based codes or PPM*. This paper takes the latter approach. The algorithm description is accompanied by a discussion of implementation methods and their relationship to the BWT-based coding techniques from which they were derived. Results given include both bounds on the code's memory and computational complexity and empirical compression performance results on the Calgary and Canterbury corpora.

The proposed algorithm is efficient in both memory and computational complexity. The algorithm guarantees worst case $O(n)$ memory and complexity in coding a sequence of length $n$, giving a significant improvement over the worst case $O\left(n^{2}\right)$ complexity of $\mathrm{PPM}^{*}$. Like $\mathrm{PPM}^{*}$, the given approach uses unbounded contexts.

The proposed algorithm gives average rates of 2.29 and $2.15 \mathrm{bpc}$ on the Calgary and Canterbury corpora, respectively. The Calgary corpus results represent a $37 \%$ decrease in per-symbol compressed file size relative to compress, a $14.9 \%$ decrease in per-symbol compressed file size relative to gzip, a $5.7 \%$ decrease in per-symbol compressed file size relative to BW94, and a $2 \%$ decrease in per-symbol compressed file size relative to PPM $^{*}$. 
The remainder of this paper is organized as follows. Section II contains a review of PPM compression algorithms. The review focuses on the $\mathrm{PPM}^{*}$ technique of Cleary et al. [2] and the exclusion-based variation of PPM* studied by Bunton [9], [10]. A short introduction to suffix trees and McCreight's efficient algorithm for suffix tree construction [19] follows in Section III. McCreight's algorithm is used in implementations of LZ [8] and BWT [12] lossless compression algorithms, but cannot be directly applied in PPM algorithms. The algorithm description is followed by a brief discussion of the difficulties inherent in applying McCreight's algorithm in PPM codes. Section IV introduces a new PPM-compatible tree construction algorithm designed specifically for use with the proposed code. Finally, the new $O(n)$ variation of the PPM* $^{*}$ algorithm is introduced in Section V. The resulting compression algorithm achieves the compression performance of PPM algorithms with the complexity of BWT and LZ codes. Section VI gives experimental results on the Calgary and Canterbury corpora and summarizes the conclusions of this work.

\section{PPM ALGORITHMS}

The lossless compression algorithms in the PPM family of codes combine sequential arithmetic source coding with adaptive Markov probability models. These system components and the methods by which they are combined are described in the discussion that follows.

Arithmetic codes appear in many lossless data compression algorithms, including both BWT and PPM codes. Part of the reason for the popularity of arithmetic codes is their low complexity. Another reason is that the arithmetic code's encoder and decoder can be applied sequentially. That is both compression and decompression can be performed symbol by symbol, as the data sequence or compressed bit stream arrives. Sequential codes yield lower delays than codes that require access to the whole data sequence or compressed bit stream before the compression or decompression process begins.

While both the PPM algorithms and the BWT-based codes rely on arithmetic coding, the PPM algorithms apply the arithmetic code directly to the original data sequence, while the BWT-based codes apply the arithmetic code to the transform output. In coding the original data sequence, the PPM algorithms maintain the sequential coding property of the arithmetic code. In contrast, BWT-based codes sacrifice the sequential coding property since the transform itself requires access to the entire data sequence.

Another reason for the popularity of arithmetic codes is their potential for achieving good lossless compression performance. Given an estimated probability $p\left(x^{n}\right)$ for an arbitrary string $x^{n}=x_{1}, \cdots, x_{n}$ of symbols from finite source alphabet $\mathcal{X}$, arithmetic coding gives a per-symbol description length $(1 / n) \ell_{n}\left(x^{n}\right)$ that satisfies $(1 / n) \ell_{n}\left(x^{n}\right)<(1 / n)\left[-\log p\left(x^{n}\right)+2\right]$ [20]. If the data string is drawn from some true underlying distribution $q$, so that the true probability of any sequence $x^{n} \in \mathcal{X}^{n}$ is $q\left(x^{n}\right)$, then (assuming an idealized coding environment) the optimal lossless source code uses $(1 / n)\left[-\log q\left(x^{n}\right)\right]$ bits per symbol in describing $x^{n}$. Thus, arithmetic codes yield excellent lossless source coding performance when provided with a good estimate $p\left(x^{n}\right)$ for the true probability $q\left(x^{n}\right)$ of data sequence $x^{n}$.

A sequential arithmetic encoder compresses each subsequent symbol $x_{i}, i \in\{1, \cdots, n\}$, using the conditional probability estimate $p\left(x_{i} \mid x^{i-1}\right)$. Likewise, the sequential decoder decompresses the binary description associated with each subsequent value $x_{i}$ using the same conditional probability model $\left\{p\left(a \mid x^{i-1}\right)\right\}_{a \in \mathcal{X}}$ used at the encoder.

The arithmetic codes in PPM algorithms rely on a collection of Markov models for forming the conditional probability estimates $\left\{p\left(a \mid x^{i-1}\right)\right\}_{a \in \mathcal{X}}$ needed to code symbol $x_{i}$. For any finite integer $k$, a $k$ th-order Markov model conditions the probability of the next symbol on only the previous $k$ symbols. Thus, an arithmetic code built using a $k$ th-order Markov model codes symbol $x_{i}$ using a conditional probability model satisfying $p\left(a \mid x^{i-1}\right)=p\left(a \mid x_{i-k}^{i-1}\right)$ for all $a \in \mathcal{X}$. In this case, the length- $k$ string $x_{i-k}^{i-1}=\left(x_{i-k}, x_{i-k+1}, \cdots, x_{i-1}\right)$ describes the context of past information on which the arithmetic code's model of the probability of symbol $x_{i}$ is conditioned.

Since the true underlying distribution $q$ is assumed not to be known a priori, the Markov models used in PPM are adaptive Markov models. The goal in adapting these models is to match the unknown distribution $q$ as closely as possible. For any symbol $a \in \mathcal{X}$ and context $s \in \mathcal{X}^{k}$, let $p_{i}(a \mid s)$ denote the adaptive Markov model's estimate at time $i$ for the conditional probability of seeing symbol $a$ given context $s$ of previous symbols. Since the encoder and decoder need access to the same conditional probability model $p_{i}(a \mid s)$ in encoding and decoding symbol $x_{i}$, an underlying constraint on the adaptation is that it must be performed in a manner that can be independently implemented at both the encoder and the decoder. While the encoder could potentially have access to all symbols $x_{1}, \cdots, x_{n}$ in coding symbol $x_{i}$, the decoder has access only to those symbols that have been previously described and successfully decoded. Thus, the conditional probability model $\left\{p_{i}(a \mid s)\right\}_{a \in \mathcal{X}}$ used to encode and decode symbol $x_{i}$ can depend only on the symbols $x_{1}, \cdots, x_{i-1}$ that are available to the sequential decoder before decoding symbol $x_{i}$.

An adaptive Markov model can update its probability estimates $p_{i}(a \mid s)$ for all $a \in \mathcal{X}$ and all $s \in \mathcal{X}^{k}$ at each time $i \in\{1, \cdots, n\}$ in order to better reflect its current estimates of the underlying source statistics. For example, $p_{i}(a \mid s)$ might be designed to track the proportion of times that the string $s$ was followed by the symbol $a$ in the first $(i-1)$ data samples $x^{i-1}$. Note that regardless of the length $k=|s|$ of context $s$, the estimate $p_{i}(a \mid s)$ is often based on information from all $(i-1)$ previously coded symbols $x^{i-1}$ in the data stream rather than just the $k$ most recently described symbols given by $s=x_{i-k}^{i-1}$.

The adaptive probability models for arithmetic codes are typically built using conditional symbol counts. For each $a \in \mathcal{X}$ and each $s \in \mathcal{X}^{k}$, conditional symbol count $N_{i}(a \mid s)$ 
describes the number of times that symbol $a$ follows string $s$ in the sequence $x_{1}, \cdots, x_{i-1}$. Thus

$$
N_{i}(a \mid s)=\sum_{j=k+1}^{i-1} 1\left(x_{j-k}^{j}=s a\right)
$$

where $1(u=v)$ denotes the indicator function, giving $1(u=v)=1$ if $u=v$ and $1(u=v)=0$ otherwise. If the probability model $\left\{p_{i}(a \mid s)\right\}_{a \in \mathcal{X}, s \in \mathcal{X}^{k}}$ relies only on the conditional symbol counts $\left\{N_{i}(a \mid s)\right\}_{a \in \mathcal{X}, s \in \mathcal{X}^{k}}$, then the decoder can track the changing probability model $p_{i}$ by keeping an independent tally of the same counts.

The source models used in PPM generalize adaptive Markov source models by replacing a single-order $k$ Markov model with a collection of Markov models of different orders. For example, given some finite memory constraint $M$, the original PPM algorithm uses Markov models of all orders $k \in\{-1,0, \cdots, M\}$, where the order $k=0$ model refers to a memoryless model and the order $k=-1$ model refers to a fixed uniform distribution on all symbols $a \in \mathcal{X}$. Typical values of $M$ for text compression satisfy $M \leq 7$ [1], [21]. PPM performance results are generally given with their corresponding memory constraints. For example, PPM5 and PPM7 designate the PPM algorithm with memory constraints $M=5$ and $M=7$ respectively.

PPM uses "escape" events to combine the prediction probabilities of its $(M+2)$ Markov models. The escape mechanism allows the encoder to tell the decoder that the symbol that follows has not previously been seen in the current context. More specifically, PPM builds its probability estimate for symbol $x_{i}$ recursively. It begins by finding the longest context of $x_{i}$ that has previously appeared in $x_{1}^{i-1}$. If symbol $x_{i}$ has not appeared in that context, then PPM describes an escape character and backs off to a shorter context. This process continues until the algorithm finds a context in which symbol $x_{i}$ is not novel, that is a context $s$ such that $N_{i}\left(x_{i} \mid s\right)>0$. (By definition, no symbol is novel in the unique context associated with $k=-1$.) Symbol $x_{i}$ is then described using that final context model.

The following definitions make these ideas precise. First, let Esc denote the escape character, where $\operatorname{Esc} \notin \mathcal{X}$, and use $\mathcal{Y}$ to denote the modified alphabet $\mathcal{Y}=\mathcal{X} \cup\{E s c\}$. Next generalize the definition of $N_{i}(a \mid s)$ from $a \in \mathcal{X}$ to $a \in \mathcal{Y}$ by defining $N_{i}(E s c \mid s)$ as

$$
N_{i}(E s c \mid s)=\sum_{a \in \mathcal{X}} 1\left(N_{i}(a \mid s)>0\right)
$$

Here, $N_{i}(E s c \mid s)$ denotes the number of distinct symbols that have followed context $s$ or, equivalently, the number of times a novel alphabet member was encountered in the given context.

Define $K(i) \leq M$ to be the length of the longest available context for symbol $x_{i}$ that has previously appeared in the given data stream. Thus, $K(1)=-1$, and for all $i>1$

$$
K(i)=\max \left\{k \leq M: x_{i-k}^{i-1}=x_{j-k}^{j-1} \wedge k+1 \leq j<i\right\} .
$$

If no context of symbol $x_{i}$ has previously appeared in the data stream, then $K(i)$ is set to 0 . The PPM algorithm uses the length- $K(i)$ context of $x_{i}$ as the initial context for the $i$ th symbol. For each $a \in \mathcal{X}$ let $k(i, a)$ be the longest context for symbol $x_{i}$ in which symbol $a$ is not novel. Thus

$$
k(i, a)=\max \left\{k: 0 \leq k \leq K(i) \wedge N_{i}\left(a \mid x_{i-k}^{i-1}\right)>0\right\} .
$$

The PPM algorithm uses index $k(i, a)$ as the length of the final context in coding symbol $x_{i}$. The final context index $k(i, a)$ is set to -1 if symbol $a$ has not previously appeared in the given data sequence. The following example demonstrates these concepts.

Example: Given data sequence $x^{6}=$ banana, the contexts for symbol $x_{7}$ are a, na, ana, nana, anana, and banana. Contexts a, na and ana have all appeared previously in the data sequence: $x_{3}$ has length- 1 context a, and $x_{5}$ has length- 1 context a, length- 2 context na, and length- 3 context ana. Context nana has not previously appeared in the data sequence. Thus, in this example, $K(7)=3$ since length- 3 context ana is the longest context of symbol $x_{7}$ that has previously appeared in the data sequence. The final context depends on the symbol $x_{7}$. If $x_{7}=\mathrm{n}$, then the final context has index $k(7, \mathrm{n})=3$ since letter $n$ is not novel in context ana. If $x_{7}=$ $\mathrm{a}$, then the final context has index $k(7, \mathrm{a})=0$ since letter a is novel in all nonzero length contexts but is not novel in the length- 0 context; that is, letter a has previously appeared in the given data stream. If $x_{7}=\mathrm{s}$, then the final context has index $k(7, \mathrm{~s})=-1$ since letter $s$ is novel in all nonzero length contexts of $x_{7}$ and has not previously appeared in the data stream.

The probability model used by PPM to code symbol $x_{i}$ is $\left\{p_{i}\left(a \mid x^{i-1}\right)\right\}_{a \in \mathcal{X}}$, where

$$
\begin{aligned}
p_{i}\left(a \mid x^{i-1}\right) & =p_{i}\left(a \mid x_{i-K(i)}^{i-1}\right) \\
& =p(i, k(i, a), a) \prod_{k=k(i, a)+1}^{K(i)} p(i, k, E s c)
\end{aligned}
$$

for each $a \in \mathcal{X}$. Here

$$
p(i, k, a)=\frac{N_{i}\left(a \mid x_{i-k}^{i-1}\right)}{\sum_{b \in \mathcal{Y}} N_{i}\left(b \mid x_{i-k}^{i-1}\right)}
$$

for each $a \in \mathcal{Y}$ and each $k \in\{0, \cdots, M\}$, while

$$
p(i,-1, a)=\frac{1}{|\mathcal{X}|}
$$

where $|\mathcal{X}|$ is the size of alphabet $\mathcal{X}$.

The above model is inefficient in its normalization of $p(i, k, a)$ for $k(i, a) \leq k<K(i)$. In particular, by describing an escape character in model $k$, the encoder tells the decoder that the observed symbol satisfies the equation $N_{i}\left(x_{i} \mid x_{i-k}^{i-1}\right)=0$. As a result, both the encoder and the decoder may exclude the possibility that the next symbol to be described equals $a$ for any $a \in \mathcal{X}$ such that $N_{i}\left(a \mid x_{i-k}^{i-1}\right)>0$. We therefore need not include any such characters in the normalization constant for distribution 
$k-1, k-2$, and so on. Modifying the normalizing constants to take this observation into account improves system performance. This modification is assumed for all $k>-1$ in the algorithm described in Section V.

The choice of a value for PPM's memory constraint $M$ is both important and difficult. The maximal memory of a natural source may not exist (that is, the source's memory may be unbounded), and even if such a bound does exist it is generally unknown. If $M$ is set to a value that is too low, then the source model is inaccurate. If $M$ is set to a value that is too high, then the source model is inefficient. Further, in practice, the best value for $M$ depends not only on the maximal memory of the true underlying source distribution $q$ but also on the data sequence length $n$. In particular, code performance suffers when $M$ is set too high, since in that case there is insufficient data to build an accurate probability model. The fact that the sequence length is typically unknown a priori further complicates the choice of $M$.

$\mathrm{PPM}^{*}$ [2] modifies PPM by removing the a priori memory constraint $M$. While the resulting code does not guarantee better performance than PPM $M$ for all possible values of $M$, the code achieves good experimental performance results across a wide variety of data types [2], [9], [10] and requires no choice of a constraint length.

In PPM*, models are kept for all contexts of all lengths that have previously appeared in the data stream under consideration. The method used to choose an initial context in PPM yields poor results for PPM*'s unbounded contexts since it allows the length of the chosen model to grow too quickly. In $\mathrm{PPM}^{*}$, Cleary et al.modify the definition of the initial context using the notion of "deterministic" contexts. A context is said to be deterministic if the number of symbols that have previously appeared in that context is exactly equal to one. In choosing its next context, PPM* uses the shortest matching deterministic context; if no such matching deterministic context exists, then $\mathrm{PPM}^{*}$ uses the longest matching context instead.

In [9] and [10], Bunton considers a collection of variations on PPM. One of those variations modifies the PPM* algorithm through the use of the "update exclusion" mechanism of [21]. The update exclusion method uses a modified symbol count $N_{i}^{\prime}(a \mid s)$ in addition to the count $N_{i}(a \mid s)$ in forming its probability estimates. While $N_{i}(a \mid s)$ increments by one each time symbol $a$ is seen in context $s \in \mathcal{X}^{*}, N_{i}^{\prime}(a \mid s)$ increments by one only if symbol $a$ is either novel in context $s$ or $s$ is a suffix of some longer context $t \in \mathcal{X}^{*}$ such that $|t|=|s|+1$ and $a$ is novel in context $t$. For each subsequent symbol $x_{i}$, the variation of PPM $^{*}$ described in [9] and [10] uses $N$ in its probability model at the initial context and $N^{\prime}$ in its probability models at all contexts reached through the escape mechanism. Thus, the resulting code must keep and update two sets of symbol counts $\{N(a \mid s)\}_{a \in \mathcal{Y}}$ and $\left\{N^{\prime}(a \mid s)\right\}_{a \in \mathcal{Y}}$ for each context $s$.

\section{SUFFIX TREES}

Maintaining the collection of probability models used by $\mathrm{PPM}^{*}$ requires keeping a record of all contexts observed in the data stream and recording the counts for symbols observed in those contexts. Both the encoder and the decoder must maintain independent records of these contexts and counts. Memory-efficient data structures and computationally efficient methods for updating them are therefore crucial for making $\mathrm{PPM}^{*}$ practical.

In $\mathrm{PPM}^{*}$, the contexts are stored in a "context trie" [2]. A context trie is a tree structure describing all contexts of all lengths that appear in a given data sequence. In its simplest form, the context trie has the single length- 0 context as its root, all previous length-1 contexts (that is, all distinct symbols that have previously appeared in the data stream) as children of the root, all length- 2 contexts (that is, all distinct length-2 patterns that have previously appeared in the data stream) descending from there, and so on. [Each length- $(k+$ 1 ) context descends from the length- $k$ context that begins with the same $k$ symbols.] For the sake of memory efficiency, context tries are often modified so that any node with only a single descendant is combined with that descendant to create a "path compressed" trie requiring fewer nodes.

Rather than growing branches of the tree for all possible contexts of all possible lengths, the context trie uses pointers to a stored copy of the original data stream. More specifically, each leaf of the context trie represents a context that has appeared exactly once in the data stream observed up to that point. The context tries of [2] include a pointer at each leaf that points to the unique location of that context in the original data stream. If a future portion of the data stream exactly matches the context stored at that leaf, then the tree is expanded by reading further into the original data stream. The path compressed tries used in $\mathrm{PPM}^{*}$ require $O(n)$ memory [2].

Like Cleary et al., Bunton stores the context information needed by PPM* in a path-compressed context trie [9], [10]. In this case, the context trie is called a "suffix tree" since every suffix of the data sequence to be compressed is represented by a path starting from the root of the tree and descending to the end-point of the data sequence. More specifically, given a data sequence $x^{n}=\left(x_{1}, \cdots, x_{n}\right)$, define the $n$ suffixes of sequence $x^{n}$ to be the strings $s_{i}=x_{i}^{n}=$ $\left(x_{i}, \cdots, x_{n}\right)$. Thus, $s_{1}=x_{1}^{n}, s_{2}=x_{2}^{n}, s_{3}=x_{3}^{n}$, and so on. The suffix tree for data sequence $x^{n}$ contains all suffixes $s_{1}, s_{2}, \cdots, s_{n}$.

Like the context-trie construction of [2], the suffix tree approach of [9] and [10] uses pointers from the leaves of the suffix tree into a stored copy of the original data stream. More traditional suffix tree descriptions, however, do not use data stream pointers at the leaves. The description that follows relies on the more traditional approach.

Using $\diamond$ to denote a unique end-of-sequence character, the suffix tree for data sequence $x^{n} \diamond$ is uniquely determined by the following three properties [19].

1) Each tree arc represents a finite nonempty string $s \in$ $\mathcal{X}^{*}$.

2) Each internal tree node except for the root has at least two children.

3) All strings represented by sibling arcs in the tree must begin with different characters.

An example of a suffix tree appears in Fig. 2. 


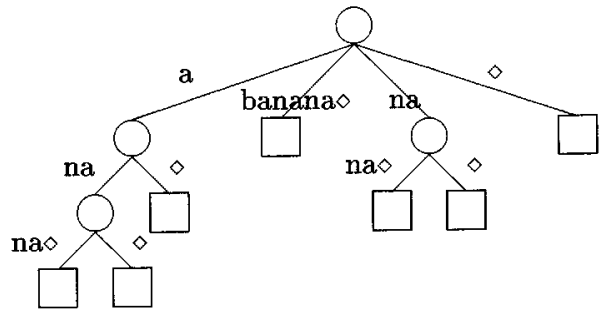

Fig. 2. A suffix tree for the sequence banana $\diamond$, where $\diamond$ denotes a unique end-of-sequence character.

A suffix tree on a sequence of length $n$ has $n$ leaves and, thus, a total of no more than $2 n$ nodes. Each arc string is stored at the node to which the given arc descends. The string description gives the string's start and end points in the original data sequence. In the implementation of [19], each node contains a single "sibling" pointer and a single "descendant" pointer to link it to the rest of the tree. The resulting tree description is linear in the sequence length $n$. Alternative data structures for suffix tree description are given in [11].

Suffix trees are useful for implementing many lossless codes. Suffix tree implementations of both LZ-style codes (e.g., [8]) and BWT-based codes [12] appear in the literature. In LZ codes, suffix trees may be used to find longest matches between the incoming data and the previously coded data. In particular, given an algorithm that adds the suffixes to the tree in order $s_{1}, s_{2}, \cdots, s_{n}$, the internal node from which suffix $s_{i}$ descends when it is first added to the tree is the longest match for data string $x_{i}, x_{i+1}, \cdots$. Since LZ codes describe the remainder of a data sequence by reference to the longest match between that data sequence and the portion of the data sequence that was previously described, and since the search in LZ codes for the longest prior match represents the complexity bottleneck for LZ code implementation, low complexity suffix tree construction algorithms are extremely useful for LZ code implementation.

Suffix trees are also useful for implementing the BWT. To understand the relationship between suffix trees and the BWT, suppose that the tree arcs descending from each node in the tree are ordered alphabetically — so branch " $a$ " falls to the left of branch "b $\diamond$ " and so on in Fig. 2. (The order imposed on the end-of-sequence symbol $\diamond$ is arbitrary. Fig. 2 treats $\diamond$ as the last letter of the alphabet.) Assuming this lexicographical ordering of tree arcs, note that the suffix tree gives a lexicographical ordering of all suffixes of the sequence $x^{n}$. In particular, each leaf of the tree represents a single suffix of $x^{n} \diamond$, and the leaves are lexicographically ordered. Given the use of a unique end-of-string symbol, the lexicographical ordering of all suffixes is equivalent to the lexicographical ordering of all cyclic shifts of the data stream. As a result, if we modify the suffix tree of Fig. 2 by marking the leaf corresponding to suffix $s_{i}=\left(x_{i}, \cdots, x_{n}\right)$ with the symbol $x_{i-1}$, then performing the BWT on $x^{n}$ is equivalent to reading off the leaf symbols from left to right through the tree.

The fact that LZ, BWT, and PPM codes can all be implemented with suffix trees points out the similarities between these seemingly very different algorithms. All three algorithms rely fundamentally on techniques for coding incoming data samples using variable-length portions of matching history. Finding these portions of matching history is central to implementing all three types of code.

Yet despite their similarities, one key property distinguishes LZ and BWT codes from PPM* . LZ and BWT codes may be implemented with linear complexity, while PPM $^{*}$ is a worst case $O\left(n^{2}\right)$ algorithm. Traditionally, the reasons for this discrepancy have been twofold. First, sequential algorithms for linear-complexity suffix-tree construction were, for many years, unknown, and unlike LZ and BWT-based codes, PPM $^{*}$ requires a sequential suffix-tree construction. Second, the probability estimation and escape mechanism used in [2] and [10] require visiting a number of contexts or probability models that is, in the worst case, quadratic in the sequence length $n$.

Traditional linear-complexity suffix tree construction algorithms like the McCreight algorithm [19] are nonsequential. In [19], suffix tree construction is performed by adding suffixes to the tree one by one in order of decreasing length. Thus, in the example of Fig. 2, the suffix "banana $\diamond$ " would be added to the tree first, followed by the suffix "anana»," followed by the suffix "nana $\diamond$ " and so on. While each subsequent suffix could be added to the tree by simply starting at the root and searching down for the longest match, the time required by this straightforward approach is super-linear. The two key observation used in [19] are:

1) the $i$ th suffix $s_{i}$ differs from the $(i+1)$ th suffix $s_{i+1}$ by only its first character $\left(s_{i}=x_{i} s_{i+1}\right)$;

2) since suffixes $s_{1}, s_{2}, \cdots$ are added to the tree in order, the longest match for suffix $s_{i}$ is known prior to the search for the longest match for suffix $s_{i+1}$.

Together, these two observations imply that if the longest match for $s_{i}$ in the previous data string is $x_{i} s$ for some $s \in$ $\mathcal{X}^{*}$, then the longest match for suffix $s_{i+1}$ must begin with string $s$. This observation allows McCreight to construct an algorithm that avoids stepping through string $s$ symbol by symbol in adding suffix $s_{i+1}$ to the suffix tree. The result is a linear-complexity algorithm. The following example illustrates the two observations described above.

Example: Given $n=6$ and data sequence $x^{6} \diamond=$ banana $\diamond$, the suffixes $s_{1}, \cdots, s_{n}$ are: $s_{1}=$ banana $\diamond, s_{2}=$ anana $\diamond, s_{3}=$ nana $\diamond, s_{4}=$ ana $\diamond, s_{5}=$ na $\diamond, s_{6}=$ a $\diamond$. Suffixes $s_{1}, s_{2}$, and $s_{3}$ have longest matches of length 0 . Thus, each suffix is given its own branch descending from the suffix tree root. Suffix $s_{4}$ finds longest match ana of length 3 by scanning from the root of the tree. In adding $s_{4}$, the branch from the root to leaf anana $\diamond$ is split into an internal node ana with two descending leaves: na $\diamond$ and $\diamond$. Using the above suffix tree properties, since the longest match for $s_{4}$ is the string ana, the longest match for $s_{5}$ must begin with the string na, and there must be a branch of the tree with a label beginning na. Knowledge of the necessary existence of such a branch makes it possible to find the branch's location without a character by character scan. In adding $s_{5}$ to the suffix tree, the node nana $\diamond$ is split into an 
internal node na with two descending leaves: na $\diamond$ and $\diamond$. Since the longest match for $s_{5}$ is the string na, the longest match for $s_{6}$ must begin with the string a. In adding $s_{6}$ to the suffix tree, the node ana $\diamond$ is split into an internal node a with two descending nodes: internal node na and leaf $\diamond$. The resulting suffix tree appears in Fig. 2.

The efficiency of McCreight's suffix tree construction technique results from its use of information about suffix $s_{i-1}$ in adding suffix $s_{i}$ to the tree, use of information about suffix $s_{i-2}$ in adding suffix $s_{i-1}$ to the tree, and so on. Since suffix $s_{1}$ equals the complete data sequence $x^{n}$, the algorithm effectively relies on knowledge of the complete data sequence in adding even the first few suffixes to the tree. As a result, construction of a suffix tree using McCreight's algorithm cannot commence until the entire data stream is known.

The reliance of McCreight's suffix tree design algorithm on knowledge of the full data sequence $x^{n}$ in adding even the first few suffixes to the tree represents a problem for PPM*. While the complete data sequence could be made available to PPM $^{*}$ 's encoder (if we assume a nonsequential code - that is a code that reads in the entire data stream before commencing the compression process), the decoder does not have access to the full data sequence during suffix tree construction. In fact, the decoder cannot decode the binary source description without access to at least part of the suffix tree. McCreight's algorithm needs the data sequence to get the suffix tree, but PPM $^{*}$ 's decoder needs the suffix tree to get the data sequence. As a result, McCreight's algorithm cannot be directly applied for suffix tree construction in PPM*.

In [22], Ukkonen introduces a linear-complexity sequential suffix-tree construction algorithm. Larsson uses that suffix-tree construction for $\mathrm{PPM}^{*}$ in [11], reducing both the complexity and the memory requirements of previous implementations of PPM $^{*}$, but noting that the final algorithm remains super-linear in complexity. The super-linear complexity of Larsson's implementation arises from the fact that the probability estimation and escape mechanism used in PPM $^{*}$ are themselves super-linear, as mentioned previously.

The next section contains an introduction to a new linearcomplexity sequential tree construction algorithm. This new alternative to Ukkonen's algorithm is designed specifically for use in PPM$^{*}$, allowing the tree construction and probabilistic modeling to be combined naturally. A linear-complexity variation of $\mathrm{PPM}^{*}$ appears in Section V.

\section{PRefix Tree Construction}

Like the variation of $\mathrm{PPM}^{*}$ given in [9] and [10], the algorithm proposed in Section $\mathrm{V}$ relies on suffix trees for computing and storing the information used in $\mathrm{PPM}^{*}$ 's probability model. Unlike the prior algorithm, however, the algorithm given here relies on a suffix tree of the reversed data stream or, equivalently, a "prefix tree" for the data stream under consideration. ${ }^{3}$ A suffix tree for the reversed

\footnotetext{
${ }^{3}$ It is important to note that the time-reversal referred to in the prefix tree algorithm is never actually implemented in practice. The given code is implemented as a prefix tree on the forward data stream rather than a suffix tree on the reversed data stream, thereby yielding a sequential algorithm.
}

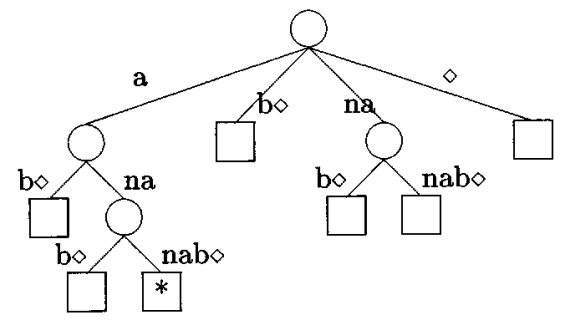

Fig. 3. A suffix tree for the sequence ananab», or, equivalently, a "prefix tree" for the sequence $\diamond$ banana.

data stream ananab $\diamond$, which is also a prefix tree for the data stream $\diamond$ banana, appears in Fig. 3. In the prefix tree, reading from the root to any leaf gives a reversed prefix. Reading leaves from left to right, the prefixes in Fig. 3 are $\diamond$ ba, $\diamond$ bana, $\diamond$ banana, $\diamond$ b, $\diamond$ ban, $\diamond$ banan, and $\diamond$.

One benefit of prefix trees is that all contexts of a given symbol lie along a single branch through the prefix tree. In particular, the longest context for symbol $x_{i}$ corresponds to the leaf labeled by $x^{i-1} \diamond$. All other contexts for symbol $x_{i}$ correspond to ancestors of that leaf. In particular, the longest context for $x_{i}$ that has previously occurred in data string $x^{i-1}$ corresponds to the parent of leaf $x^{i-1}$. The following example illustrates this property.

Example: Suppose that we are constructing a prefix tree as we code each subsequent symbol of a data string $x^{n}$, and suppose that the symbols seen so far are $x^{i-1}=$ banana. Then the prefix tree available for coding symbol $x_{i}$ is the prefix tree of Fig. 3. The contexts for symbol $x_{i}$ are: a, na, ana, nana, anana, and banana. All of these contexts are represented along the single branch going from the root of the tree to the leaf marked ${ }^{*}$ in Fig. 3 . The reversed contexts (a, an, ana, anan, anana, and ananab) label the partial substrings of this branch, with $\diamond$ appended to the longest context to mark it as a complete prefix of string $x^{n}$. The longest matching context for symbol $x_{i}$ is the context ana corresponding to the internal node from which leaf $x^{i-1}=\diamond$ banana descends.

The next step is to devise a low complexity sequential prefix tree construction algorithm that can be employed within codes like PPM*. The order in which PPM* $^{*}$ makes prefixes of the data string available for addition to the prefix tree (or suffixes of the reversed data string available for addition to the suffix tree) is exactly opposite the order in which strings are added to the tree in McCreight's algorithm. More specifically, in PPM*, symbols become available to the decoder sequentially, where symbol $x_{i}$ can be decoded only after the context tree based on symbols $x_{1}, \cdots, x_{i-1}$ has been constructed. Thus, using $p_{i}=\left(x_{1}, \cdots, x_{i}\right)$ to denote the $i$ th prefix of string $x^{n}, \mathrm{PPM}^{*}$ requires an algorithm for adding prefixes in order from shortest to longest - that is order $p_{1}=\left(x_{1}\right), p_{2}=\left(x_{1}, x_{2}\right), \cdots, p_{n}=\left(x_{1}, \cdots, x_{n}\right)$. In contrast, McCreight's algorithm applied to the reversed data stream adds the longest prefix of the data stream (or the longest suffix of the reversed data stream) first and then follows in order of decreasing length-giving order $p_{n}=s_{1}, p_{n-1}=s_{2}, \cdots, p_{1}=s_{n}$.

Since reading from root to leaf along a single path through a prefix tree gives the reversed prefix, finding the right place 
to add a new prefix to an existing prefix tree is equivalent to finding the longest matching suffix of the given prefix. The complexity bottleneck in building prefix trees is the search for this longest match for each subsequent prefix $p_{i}$. While McCreight's algorithm cannot be directly applied here, the basic observations on which that algorithm is built remain valid. The following observations are the logical analogies for those used by McCreight when order $p_{n}, p_{n-1}, \cdots, p_{1}$ is replaced by order $p_{1}, p_{2}, \cdots, p_{n}$.

1) Prefix $p_{i}$ differs from prefix $p_{i-1}$ by only its last character; that is, $p_{i}=p_{i-1} x_{i}$. As a result, the longest match for prefix $p_{i}$ here denoted by $\operatorname{suf}_{i}\left(p_{i}\right)$, is at most one character longer than the longest match for prefix $p_{i-1}$ and can rely on no characters prior to those found in the longest match for prefix $p_{i-1}$. More precisely

$$
\operatorname{suf}_{i}\left(p_{i}\right)=\operatorname{suf}_{i}\left(p_{i-1} x_{i}\right)=\operatorname{suf}_{i}\left(\operatorname{suf}_{i-1}\left(p_{i-1}\right) x_{i}\right)
$$

2) Since the prefixes are added to the tree in order $p_{1}, p_{2}, \cdots, p_{n}$, the longest match for prefix $p_{i-1}$ is known prior to the search for the longest match for prefix $p_{i}$.

To exploit these observations, and thereby decrease the complexity associated with searching for the longest match for each subsequent prefix, the proposed prefix tree construction algorithm adds auxiliary links to the data structure. These links provide "short-cuts" in the search for the longest match. A description of the algorithm follows.

The tree is initialized to a single node corresponding to the length-0 (empty) string. The initial tree is labeled $T_{0}$. The prefix tree is then built up one leaf at a time, by adding one prefix at each time step. After the $(i-1)$ th addition, the tree contains prefixes $p_{1}, p_{2}, \cdots, p_{i-1}$ and is called $T_{i-1}$. Associated with every node in the tree except for the most recently added leaf $\left(p_{i-1}\right)$ is a nonempty array of "short-cut" pointers. The number of elements in a short-cut array equals the number of symbols seen so far in the corresponding context. The short-cut pointer for symbol $a$ located at the tree node for context $s$ points to the tree node for context $s a$ (or some extension $s^{\prime} s a$ of $s a$ if there exists some string $s^{\prime} \in \mathcal{X}^{*}$ such that all previous appearances of $s a$ were preceded by string $s^{\prime}$ ) and is created the first time symbol $a$ appears in context $s$.

Recall that $\operatorname{suf}_{i-1}\left(p_{i-1}\right)$ is the longest match for prefix $p_{i-1}$ that has previously appeared in the data stream. Step $i$ of the tree construction algorithm begins at node $\operatorname{suf}_{i-1}\left(p_{i-1}\right)$ and ends at node $\operatorname{suf}_{i}\left(p_{i}\right)$. [Here, $\operatorname{suf}_{0}\left(p_{0}\right)$ is defined to be the root of the empty tree $T_{0}$.] If symbol $x_{i}$ has previously appeared in context $\operatorname{suf}_{i-1}\left(p_{i-1}\right)$, then $\operatorname{suf}_{i-1}\left(p_{i-1}\right)$ has a short-cut pointer for symbol $x_{i}$. Traversing this pointer leads to node $\operatorname{suf}_{i}\left(p_{i}\right)=\operatorname{suf}_{i-1}\left(p_{i-1}\right) x_{i}$. If $x_{i}$ has not previously appeared in context $\operatorname{suf}_{i-1}\left(p_{i-1}\right)$, then the algorithm moves to the parent of the given node and looks for a short-cut pointer there. This procedure continues until the algorithm either finds and traverses a short-cut pointer or ends up at the root.

The final node in the above procedure is either $\operatorname{suf}_{i}\left(p_{i}\right)$ or an extension of $\operatorname{suf}_{i}\left(p_{i}\right)$. If the node reached corresponds to $\operatorname{suf}_{i}\left(p_{i}\right)$, then a single leaf is added to the tree; the added leaf descends from $\operatorname{suf}_{i}\left(p_{i}\right)$ and represents prefix $p_{i}$. If the node reached corresponds to an extension of $\operatorname{suf}_{i}\left(p_{i}\right)$, then both an internal node and a leaf are added to the tree. The internal node descends from the previous parent of the extension node and has two children: one is the extension node itself, and the other is the leaf for $p_{i}$. All contexts of $x_{i}$ visited in the unsuccessful portion of the search for a matching shortcut pointer are given short-cut pointers pointing to the new leaf $p_{i}$.

If building tree $T_{i}$ from tree $T_{i-1}$ required the addition of a new internal node, the shortcut pointers that previously pointed to the extension of $\operatorname{suf}_{i}\left(p_{i}\right)$ may require updating. In particular, shortcut pointers that previously pointed to the $e x$ tension of $\operatorname{suf}_{i}\left(p_{i}\right)$ for which the newly added internal node $\operatorname{suf}_{i}\left(p_{i}\right)$ is either the desired shortcut destination or an extension of the desired shortcut destination are updated to point to the new internal node $\operatorname{suf}_{i}\left(p_{i}\right)$.

To calculate the complexity of the proposed tree construction algorithm, let $k_{i}$ denote the number of contexts of $x_{i}$ visited in searching for $\operatorname{suf}_{i}\left(p_{i}\right)$. Finding the complexity of the tree construction algorithm is equivalent to finding $\sum_{i=1}^{n} k_{i}$. Repeated application of the equality $\left|\operatorname{suf}_{i}\left(p_{i}\right)\right|=\left|\operatorname{suf}_{i-1}\left(p_{i-1}\right) x_{i}\right|-k_{i}$ accomplishes that goal, giving finally $\sum_{i=1}^{n} k_{i}=n-\left|\operatorname{suf}_{n}\left(p_{n}\right)\right| \leq n$. Thus, in total at most $n$ nodes must be visited in the search for the longest matches for prefixes $p_{1}, \cdots, p_{n}$.

\section{AN $O(n)$ PPM* AlgORITHM $^{*}$}

The proposed prefix tree construction algorithm can be employed in any environment where sequential prefix or suffix tree construction is desired. For example, the given algorithm can be applied in place of McCreight's algorithm in both LZ and BWT-based codes. The proposed prefix tree construction is especially well suited for use in the PPM* algorithm since:

1) the memory needs of the tree construction algorithm combine naturally with those of the PPM* algorithm;

2) the nodes visited in constructing the tree must also be visited in calculating PPM*'s probability models.

These points are treated in turn below.

As in [9]-[11], the use of prefix (or, equivalently, suffix) trees yields a space-efficient means for storing PPM*'s probability models. In particular, the proposed algorithm is $O(n)$ in memory. As in [11], the counts used in the probability model are stored in the nodes to which they correspond. The short-cut pointers of the proposed approach replace the suffix pointers of the earlier approach.

The proposed tree construction algorithm combines naturally with $\mathrm{PPM}^{*}$ not only in the related storage needs of the two algorithms but also in the nodes visited. In particular, the tree construction process of visiting shorter and shorter contexts until finding a short-cut pointer for symbol $x_{i}$ corresponds exactly with the PPM* coding process of visiting Markov models with shorter and shorter contexts until finding a model in which symbol $x_{i}$ is not novel. As a result, these functions are naturally combined in implementing the proposed algorithm. Further, since the collection of nodes visited corresponds exactly to the set of contexts $s$ for which 
either symbol $x_{i}$ is novel in context $s$ or $s$ is a suffix of some longer context $t \in \mathcal{X}^{*}$ such that $|t|=|s|+1$ and symbol $x_{i}$ is novel in context $t$, updating the counts $N_{i}^{\prime}\left(x_{i} \mid s\right)$ can likewise be combined with the prefix tree construction.

Unfortunately, as in [11], direct application of the above $O(n)$ tree construction algorithm in place of a worst case $O\left(n^{2}\right)$ tree construction algorithm in either the original $\mathrm{PPM}^{*}$ algorithm of [2] or the variation of $\mathrm{PPM}^{*}$ described in [9] and [10] fails to yield an $O(n)$ compression algorithm. The problem arises from the fact that keeping accurate models of $N_{i}(a \mid s)$ and looking for the shortest deterministic context for symbol $x_{i}$ both require (worst case) $O\left(n^{2}\right)$ calculations. The following example illustrates these worst case complexity results.

Example: Consider a string $x^{n}=$ aaaaa $\cdots$ aaaa. In this example, the length of the longest matching context for symbol $x_{i}$ is $(i-2)$ for each $i \geq 2$. As a result, after seeing symbol $x_{i}, N_{i}\left(x_{i} \mid s\right)$ must be updated for $(i-2)$ contexts $s$. Since $\sum_{i=2}^{n}(i-2)$ grows as $n^{2}$, the number of nodes visited and the number of operations performed are both $O\left(n^{2}\right)$. Further, since every context in this example is a deterministic context, the number of nodes visited in starting at the longest matching context and searching for the shortest deterministic context is likewise $O\left(n^{2}\right)$.

Since the symbol counts $N_{i}(a \mid s)$ are used in all probability models in [2] and in the probability models for all initial contexts in [9] and [10], and since both algorithms employ the shortest deterministic context as the starting point for their probability models, these algorithms cannot achieve the desired worst case $O(n)$ complexity.

We next modify the PPM $^{*}$ algorithm in order to guarantee worst case $O(n)$ complexity. The proposed modifications are very simple. First, we rely entirely on $N_{i}^{\prime}(a \mid s)$ rather than the combination of $N_{i}^{\prime}(a \mid s)$ and $N_{i}(a \mid s)$ used in [9] and [10]. Second, we put a fixed cap on the number of times (per symbol $x_{i}$ ) the algorithm can move to a shorter deterministic context. (The experiments reported in the next section set this cap at 16.) Each of these choices comes at the expense of some penalty in compression performance. Fortunately, experimental results indicate that the penalty is generally quite small, while the savings in complexity (and, thus, run-time) is large.

\section{RESULTS AND CONCLUSION}

This paper introduces a new variation of $\mathrm{PPM}^{*}$. By altering the tree structure used to store the code's probability models, the construction algorithm used to sequentially build that tree, and the probability models themselves, the proposed algorithm reduces the worst case $O\left(n^{2}\right)$ complexity of PPM* to worst case $O(n)$ complexity. The new algorithm maintains the $O(n)$ memory of earlier PPM* algorithms. Tables 1 and 2 show the rate results achieved by the proposed algorithm (labeled as "new") as compared to those of a variety of alternative algorithms. The results for competing algorithms on the Calgary corpus are quoted from [2] (labeled $\mathrm{PPM}^{*}$ ) and [9] and [10] (labeled B97). The results on the Canterbury corpus are quoted from the Canterbury corpus web page.
Table 1

Compression Results on the Calgary Corpus

\begin{tabular}{l||c|c|c|c}
\hline \multicolumn{5}{c}{ Calgary Corpus } \\
\hline \hline File & $\begin{array}{c}\text { B97 } \\
\text { (bpc) }\end{array}$ & $\begin{array}{c}\text { NEW } \\
\text { (bpc) }\end{array}$ & $\begin{array}{c}\text { PPM }^{*} \\
\text { (bpc) }\end{array}$ & $\begin{array}{c}\text { BW94 } \\
\text { (bpc) }\end{array}$ \\
\hline \hline bib & 1.79 & 1.87 & 1.91 & 2.07 \\
bk1 & 2.18 & 2.44 & 2.40 & 2.49 \\
bk2 & 1.86 & 2.02 & 2.02 & 2.13 \\
geo & 4.46 & 4.71 & 4.83 & 4.45 \\
news & 2.29 & 2.38 & 2.42 & 2.59 \\
obj1 & 3.68 & 3.75 & 4.00 & 3.98 \\
obj2 & 2.28 & 2.33 & 2.43 & 2.64 \\
ppr1 & 2.25 & 2.34 & 2.37 & 2.55 \\
ppr2 & 2.21 & 2.34 & 2.36 & 2.51 \\
pic & 0.78 & 0.88 & 0.85 & 0.83 \\
prgc & 2.29 & 2.35 & 2.40 & 2.58 \\
prgl & 1.55 & 1.61 & 1.67 & 1.80 \\
prgp & 1.53 & 1.58 & 1.62 & 1.79 \\
trns & 1.33 & 1.40 & 1.45 & 1.57 \\
\hline \hline AVG & 2.18 & 2.29 & 2.34 & 2.43 \\
\hline \multicolumn{4}{|c|}{}
\end{tabular}

Table 2

Compression Results on the Canterbury Corpus

\begin{tabular}{l||c|c|c|c}
\hline \multicolumn{5}{c}{ Canterbury Corpus } \\
\hline \hline File & $\begin{array}{c}\text { PPMZ9 } \\
(\mathbf{b p c})\end{array}$ & $\begin{array}{c}\text { NEW } \\
(\mathrm{bpc})\end{array}$ & $\begin{array}{c}\text { PPM7 } \\
(\mathrm{bpc})\end{array}$ & $\begin{array}{c}\text { BZIP2 } \\
(\mathrm{bpc})\end{array}$ \\
\hline \hline text & 2.08 & 2.22 & 2.26 & 2.27 \\
fax & 0.79 & 0.88 & 0.94 & 0.78 \\
csrc & 1.87 & 1.96 & 2.08 & 2.18 \\
excl & 1.01 & 1.40 & 0.97 & 1.01 \\
sprc & 2.45 & 2.61 & 2.58 & 2.70 \\
tech & 1.83 & 1.99 & 2.01 & 2.02 \\
poem & 2.22 & 2.45 & 2.46 & 2.42 \\
html & 2.19 & 2.30 & 2.35 & 2.48 \\
lisp & 2.25 & 2.35 & 2.43 & 2.79 \\
man & 2.87 & 2.95 & 3.01 & 3.33 \\
play & 2.34 & 2.52 & 2.55 & 2.53 \\
\hline \hline AVG & 1.99 & 2.15 & 2.15 & 2.23 \\
\hline
\end{tabular}

While the results given in Tables 1 and 2 are by no means exhaustive, they give a reasonable picture of how the performance of the proposed algorithm compares to current alternatives. The average results on the Calgary corpus give a $37 \%$ decrease in per-symbol compressed file size relative to compress, a $14.9 \%$ decrease in per-symbol compressed file size relative to gzip, a $5.7 \%$ decrease in per-symbol compressed file size relative to BW94, and a $2 \%$ decrease in per-symbol compressed file size relative to PPM* The average results on the Canterbury corpus give a $35.5 \%$ decrease in per-symbol compressed file size relative to compress, a $15 \%$ decrease in per-symbol compressed file size relative to gzip, a 3.6\% decrease in per-symbol compressed file size relative to BZIP2 (a popular BWT-based algorithm), and the same per-symbol compressed file size as PPM7. While the proposed approach is not the best known algorithm in rate performance, it gives a very good tradeoff in effectiveness and efficiency - surpassing both PPM* and the BWT-based codes in compression capabilities using only $O(n)$ memory and complexity. In short, the proposed algorithm achieves the performance of a PPM algorithm using the complexity of a BWT-based code. 


\section{REFERENCES}

[1] J. G. Cleary and I. H. Witten, "Data compression using adaptive coding and partial string matching," IEEE Trans. Commun., vol. 32, pp. 396-402, Apr. 1984.

[2] J. G. Cleary, W. J. Teahan, and I. H. Witten, "Unbounded length contexts for PPM," in Proc. Data Compression Conf. Snowbird, UT: IEEE, Mar. 1995, pp. 52-61.

[3] T. C. Bell, J. G. Cleary, and I. H. Witten, Text Compression. Englewood Cliffs, NJ: Prentice-Hall, 1990

[4] R. Arnold and T. Bell, "A corpus for the evaluation of lossless compression algorithms," in Proc. Data Compression Conf. Snowbird, UT: IEEE, Mar. 1997, pp. 201-210.

[5] J. Ziv and A. Lempel, "A universal algorithm for sequential data compression," IEEE Trans. Inform. Theory, vol. IT-23, pp. 337-343, May 1977.

[6] - "Compression of individual sequences via variable-rate coding," IEEE Trans. Inform. Theory, vol. IT-24, pp. 530-536, Sept. 1978.

[7] T. A. Welch, "A technique for high-performance data compression," IEEE Comput., vol. 17, pp. 8-19, June 1984.

[8] M. Rodeh, V. R. Pratt, and S. Even, "Linear algorithm for data compression via string matching," J. Assoc. Comput. Mach., vol. 28, pp. 16-24, Jan. 1981.

[9] S. Bunton, "On-line stochastic processes in data compression," $\mathrm{Ph} . D$. dissertation, Univ. Washington, Seattle, WA, 1996.

[10] - "Semantically motivated improvements for PPM variants," Comput. J., vol. 40, no. 2/3, pp. 76-93, 1997.

[11] N. J. Larsson, "Structures of string matching and data compression," Ph.D. dissertation, Lund Univ., Lund, Sweden, 1999.

[12] M. Burrows and D. J. Wheeler, "A block-sorting lossless data compression algorithm," Digital Systems Research Center, Palo Alto, CA, Tech. Rep. SRC 124, May 1994.

[13] Z. Arnavut and S. S. Magliveras, "Lexical permutation sorting algorithm," Comput. J., vol. 40, no. 5, pp. 292-295, 1997.

[14] N. J. Larsson, "The context trees of block sorting compression," in Proc. Data Compression Conf. Snowbird, UT: IEEE, Mar. 1998, pp. $189-198$.

[15] M. Effros, "Universal lossless source coding with the Burrows Wheeler transform," in Proc. Data Compression Conf. Snowbird, UT: IEEE, Mar. 1999, pp. 178-187.

[16] _ - "Universal lossless source coding with the Burrows Wheeler transform," IEEE Trans. Inform. Theory, June 1999, submitted for publication.

[17] _ "Theory meets practice: Universal source coding with the Burrows Wheeler transform," in Proc. Allerton Conf. Communication, Control, and Computing. Monticello, IL: IEEE, Sept. 1999.
[18] — "PPM performance with BWT complexity: A new algorithm for lossless data compression," in Proc. Data Compression Conf., Snowbird, UT, Mar. 2000.

[19] E. M. McCreight, "A space-economical suffix tree construction algorithm," J. ACM, vol. 23, pp. 262-272, Apr. 1976.

[20] F. Jelinek, "Buffer overflow in variable length coding of fixed rate sources," IEEE Trans. Inform. Theory, vol. 14, pp. 490-501, May 1968.

[21] A. Moffat, "Implementating the PPM data compression scheme," IEEE Trans. Commun., vol. 38, pp. 1917-1921, Nov. 1990.

[22] E. Ukkonen, "On-line construction of suffix trees," Algorithmica, vol. 14 , no. 3, pp. 249-260, 1995

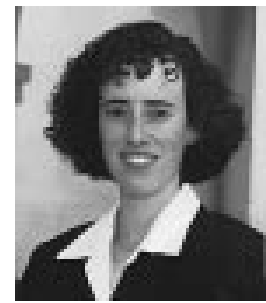

Michelle Effros received the B.S. degree with distinction in 1989, the M.S. degree in 1990, and the Ph.D. degree in 1994, all in electrical engineering, from Stanford University, Stanford, CA.

During the summers of 1988 and 1989, she worked at Hughes Aircraft Company, researching modulation schemes, real-time implementations of fast data rate error correction schemes, and future applications for fiber optics in space technology. Since 1994, she has been with the

Department of Electrical Engineering, California Institute of Technology, Pasadena, where she is currently an Associate Professor. Her research interests include information theory, data compression, communications, pattern recognition, speech recognition, and image processing.

Dr. Effros received Stanford's Frederick Emmons Terman Engineering Scholastic Award (for excellence in engineering) in 1989, the Hughes Masters Full-Study Fellowship in 1989, the National Science Foundation Graduate Fellowship in 1990, the AT\&T Ph.D. Scholarship in 1993, the NSF CAREER Award in 1995, the Charles Lee Powell Foundation Award in 1997, the Richard Feynman-Hughes Fellowship in 1997, and the Okawa Foundation Research Grant in 2000. She is a Member of Tau Beta Pi, Phi Beta Kappa, Sigma Xi, and the IEEE Information Theory, Signal Processing, and Communications societies. She served as the Editor of the IEEE Information Theory Society Newsletter from 1995 to 1998 and has been a Member of the Board of Governors of the IEEE Information Theory Society since 1998. 